\title{
A Calculation Procedure for Two-Dimen- sional Parabolic Two-Phase Flows Using Independent Computational Grids for Each Phase*
}

\author{
Daniel FLECKHAUS**, Koichi HISHIDA** \\ and Masanobu MAEDA**
}

\begin{abstract}
A computational marching procedure for two-phase flows which are dispersed, parabolic, and turbulent is outlined in detail. Convection, diffusion and viscous action as well as entrainment and volume fraction fluctuations are taken into account for both phases. Each phase is calculated in its own grid to reduce false diffusion to a minimum. The use of two independent grids requires formulae for the transfer of variables which are presented. A test case is examined to estimate the effect of false diffusion on the second phase. A two-phase jet is calculated and the results are found to be in good agreement with experimental data.
\end{abstract}

Key Words: Fluid Mechanics, Gas-Solid Two-Phase Flow, Parabolized Continuum Equations, Numerical Calculation, Finite Difference Method

List of Symbols
$A_{i}:$ cross stream area
$a_{i}:$ coefficient in Eq. (14)
$b_{i}:$ coefficient in Eq. (14)
$c_{i}:$ coefficient in Eq. (14)
$C:$ constant
$D$ : outlet diameter
$d_{i}:$ coefficient
$d_{p}:$ particle diameter
$F:$ interphase friction factor
$k:$ kinetic energy
$M:$ mass loading ratio
$\dot{M}^{\prime}$ : lateral mass flow
$N$ : number density
$N_{c}$ : number density at the center line
$p:$ lateral pressure
$P$ : axial pressure
$S:$ source term
$U$ : mean velocity in main stream direction

* Received 27th October, 1986. Paper No. 86-2009 B

** Department of Mechanical Engineering, Keio University, 3-14-1 Hiyoshi, Kohoku-ku, Yokohama, 223, Japan $u^{\prime}$ : velocity fluctuations of $U$

$V:$ mean velocity in lateral direction

$v^{\prime}$ : velocity fluctuations of $V$

$X$ : coordinate in main stream direction

$Y$ : coordinate in lateral direction

\section{Greek Letters}

$\alpha$ : volume fraction first phase

$\alpha^{\prime}$ : volume fraction fluctuation of $\alpha$

$\beta$ : volume fraction second phase

$\beta^{\prime}$ : volume fraction fluctuation of $\beta$

$\gamma:$ volume fraction

$\gamma^{\prime}:$ volume fraction fluctuation

$\varepsilon:$ dissipation rate

$\zeta:$ angle of stream lines

$\nu:$ turbulence viscosity

$\xi$ : angle of $\omega$ - lines

$\rho:$ density

$\sigma:$ Schmidt number

$\phi:$ general variable

$\psi:$ stream function

$\omega:$ transformed lateral coordinate

$\Omega: \omega$-interval

Indices

$E$ : outer boundary 


$$
\begin{aligned}
& f: \text { first phase } \\
& I: \text { inner boundary } \\
& s: \text { second phase } \\
& U: \text { upstream } \\
& \phi: \text { general variable }
\end{aligned}
$$

\section{Introduction}

Successful calculation procedures for parabolic single-phase flows are well known ${ }^{(1)(2)}$ and have been applied to a wide range of fluid problems ${ }^{(3)}$. The main feature of these procedures is the marching technique which requires the momentary storage of variables for only one step in the main stream direction. This feature enables the effective use of computer storage, and within acceptable costs allows the use of a fine grid to obtain grid independent solutions. The resulting accuracy is highly desired when comparing numerical predictions with experimental results and evaluating physical models.

For multi-phase flows, the computational expenses increase considerably. Not only more variables have to be stored, but also a greater number of differential equations have to be solved, slowing down the convergence of a solution procedure. This increases the expenditure making a procedure with a marching technique even more attractive for parabolic multi-phase flows. Spalding ${ }^{(4)}$ developed a computational code for two-phase flows. The flow had to be fully dispersed and parabolic. Although this code is suitable for many applications, some objections have to be made.

First, the differential equations incorporated for the second phase are more than necessarily simplified. Convection, diffusion, and viscous action are neglected, which is not always permissible. Mass entrainment of the second phase is not accounted for and volume fraction fluctuations are neglected for both phases, although they are part of the continuity equations and thus part of the convection terms of other continuum equations. Second, the velocity vector of the second phase is usually not closely aligned with the mesh line of the first phase which can lead to an undesirable amount of false diffusion.

A reduction of false diffusion is often possible only by using an excessively fine grid or by implementing a scheme which takes account of the velocity vector. The former will lead to a considerable increase in expenditure and the latter may add convergence problems.

An alternative is to chose a separate grid for the second phase which ensures that the velocity vector is aligned with this grid, or is at least very close, depending on the entrainment of the second phase. This allows the use of even a simple conventional scheme (for example, the hybrid scheme) to obtain accurate results. The additional efforts which have to be made are to find an appropriate and easy-to-compute way of exchanging variables between the grid of the first phase and the grid of the second phase.

The purpose of this paper is to outline a computational procedure for a two-phase flow which is dispersed, parabolic, and turbulent. Convection, diffusion and viscous action as well as entrainment and volume fraction fluctuations are taken into account for both phases. Each phase is calculated in its own grid to reduce false diffusion to a minimum. Two independent grids require a method of variable exchange, which is presented in detail. An extreme case is examined to estimate the effect of false diffusion on the second phase. A two-phase jet is calculated and compared to experimental data.

\section{Equations and Calculation Procedure}

\section{1 Continuum equations}

Separate conservation equations are formulated for each phase using the continuum approach for a control volume analysis. To obtain equations for a turbulent flow, a conventional Reynolds decomposition is performed replacing randomly changing flow variables by time averages plus fluctuations about the average. It may be written as $\tilde{U}=U+u^{\prime}, \tilde{V}=V+v^{\prime}, \tilde{\gamma}$ $=\gamma+\gamma^{\prime} \cdots$. All terms containing only one fluctuating quantity as a parameter vanish after an integration over a sufficient large time period, leading to a set of general conservation equations which have been presented by Sha and Soo ${ }^{(5)}$. Although these equations are suitable for describing a broad range of fluid flows, they are sometimes over specific and are therefore inefficient. One of these cases is a steady two-dimensional flow which satisfies the following conditions:

The gradients in the streamwise direction are much smaller than those in the cross stream direction

$\frac{\partial}{\partial X} \ll \frac{\partial}{\partial Y}$.

The variation of pressure in the streamwise and normal direction is decoupled

$\bar{P}(X, Y)=P(X)+p(Y)$.

There is no upstream-pressure-effect which requires that

$P(X) \ll \frac{1}{2} \rho U_{r e f}^{2}$

where $U_{\text {ref }}$ is a suitably defined reference velocity (e.g. free stream velocity).

These requirements are sometimes referred to as the 'thin shear layer assumptions', although the flow in question is not necessarily a 'thin' shear layer since the assumptions are also valid for quite extended 
flows like free jets and others. It is obvious that diffusive fluxes in the streamwise direction are negligible under the first condition, which leads to a considerable simplification of the continuum equations. The equations become parabolized ${ }^{(6)}$. The averaged, steady state conservation equations for the mass and momentum of a two-phase flow can then be written as follows :

Continuity

$$
\frac{\partial}{\partial X}(\rho \gamma U)+\frac{\partial}{\partial Y}\left(\rho \gamma V+\rho \overline{\gamma^{\prime} v^{\prime}}\right)=0 .
$$

Momentum on the main stream in the $X$-direction

$$
\begin{aligned}
& \frac{\partial}{\partial X}(\rho \gamma U U)+\frac{\partial}{\partial Y}\left[U\left(\rho \gamma V+\rho \overline{\gamma^{\prime} v^{\prime}}\right)\right] \\
& =-\frac{\partial}{\partial Y}\left(\rho \gamma \overline{v^{\prime} u^{\prime}}+\rho \overline{\gamma^{\prime} v^{\prime} u^{\prime}}\right) \\
& -\gamma \frac{d P}{d X}-F \beta\left(U-U^{*}\right)+S_{x} .
\end{aligned}
$$

Momentum in the lateral $Y$-direction

$$
\begin{aligned}
& \frac{\partial}{\partial X}(\rho \gamma U V)+\frac{\partial}{\partial Y}\left[V\left(\rho \gamma V+\rho \overline{\gamma^{\prime} v^{\prime}}\right)\right] \\
& \quad=-\frac{\partial}{\partial Y}\left(\rho \gamma \overline{v^{\prime} v^{\prime}}+\rho V \overline{\gamma^{\prime} v^{\prime}}+\rho \overline{\gamma^{\prime} v^{\prime} v^{\prime}}\right) \\
& -\gamma \frac{d p}{d Y}-F \beta\left(V-V^{*}\right)-F \overline{\beta^{\prime}\left(\overline{v^{\prime}-v^{* \prime}}\right)}+S_{y} .
\end{aligned}
$$

Finally, the global continuity equation gives $\alpha+\beta=1$.

Equations ( 1 and 3 ) are expressed in a general form and are valid for both phases. The equations for the first-phase are obtained if $\gamma$ is replaced by $\alpha$, and $U^{*}, V^{*}$ by the second-phase $U$ - and $V$ - velocities. The axial pressure $P$ and the lateral pressure $p$ as well as the interphase friction factor $F$ are shared by both phases. The variable $\beta$ always represents the volume fraction of the second-phase. For the equations of the second-phase, $\gamma$ has to be replaced by $\beta$, and $U^{*}, V^{*}$ by the first-phase $U$ - and $V$ - velocities. The sources $S_{x}$ and $S_{y}$ may contain forces like gravity or extra body forces, and may not necessarily be equal for both phases. The interphase friction coefficient $F$ is a function of the relative difference between the first-and second-phase velocity ${ }^{(7)}$.

In addition to the already introduced differential equations, other properties have to be calculated, such as the kinetic energy $k$, its dissipation rate $\varepsilon$, temperatures and others. These passive scalars are governed by a type of equation which is of the form

$$
\begin{aligned}
& \frac{\partial}{\partial X}(\rho \gamma U \phi)+\frac{\partial}{\partial Y}\left[\left(\rho \gamma V+\rho \overline{\gamma^{\prime}} \overline{v^{\prime}}\right) \phi\right] \\
& =-\frac{\partial}{\partial Y}\left(\rho \gamma \overline{v^{\prime} \phi^{\prime}}\right)+S_{\phi} .
\end{aligned}
$$

A comparison with the momentum equations reveals that Eq. ( 5 ) is also a general representation of the momentum equations, Eqs. ( 2 ) and ( 3 ). It is about this generality that for all variables only one solution procedure is necessary. The continuity equation, Eq. (1), has also the form of Eq. ( 5 ) but will be treated differently, as outlined in chapter 2.4 .

\section{2 Fluctuation terms}

The above conservation equations contain several fluctuation terms which have to be modeled. Using the Boussinesq assumption, the Reynolds stresses are

$$
\overline{v_{i}^{\prime} \bar{v}_{j}^{\prime}}=-\nu\left(\frac{\partial U_{i}}{\partial X_{j}}+\frac{\partial U_{j}}{\partial X_{i}}\right)+\frac{2}{3} \delta_{i j}\left(\nu \frac{\partial U_{k}}{\partial X_{k}}+k\right)(6)
$$

and are parabolized as

$$
\begin{aligned}
& \overline{v^{\prime} u^{\prime}}=-\nu \frac{\partial U}{\partial Y} \\
& \overline{v^{\prime} v^{\prime}}=-\nu\left(\frac{\partial V}{\partial Y}+\frac{\partial V}{\partial Y}\right)+\frac{2}{3} \nu \frac{\partial Y}{\partial V}=-\frac{4}{3} \nu \frac{\partial V}{\partial Y} .
\end{aligned}
$$

For the terms containing passive scalars like $\overline{\gamma^{\prime} v^{\prime}}$ and $\overline{v^{\prime} \overline{\phi^{\prime}}}$ a gradient transport term is assumed:

$$
\overline{v^{\prime} \phi^{\prime}}=-\frac{\nu}{\sigma_{\phi}} \frac{\partial \phi}{\partial Y}
$$

with a Schmidt number $\sigma_{\phi}$.

The third order term $\overline{r^{\prime} v^{\prime} u^{\prime}}$ can be modeled as ${ }^{(7)}$

$$
\overline{\gamma^{\prime} u_{i}^{\prime} u_{j}^{\prime}}=-C_{\phi}\left(\frac{k}{\varepsilon}\right)\left(\overline{u_{i}^{\prime} u_{k}^{\prime}} \frac{\partial \overline{u_{j}^{\prime} \gamma^{\prime}}}{\partial X_{k}}\right)
$$

and parabolized as

$$
\overline{\gamma^{\prime} v^{\prime} u^{\prime}}=-C_{\phi} \frac{k}{\varepsilon} \overline{u^{\prime} v^{\prime}} \frac{\partial \overline{\gamma^{\prime} v^{\prime}}}{\partial Y}
$$

or may simply be neglected due to its small order of magnitude. The fluctuation term $\overline{\beta^{\prime}\left(u^{\prime}-u^{* \prime}\right)}$ can be rewritten. After the Reynolds decomposition but before the time averaging, the global continuity equation yields $\alpha^{\prime}=-\beta^{\prime}$. This gives two expressions for the above term equivalent to Eq. ( 8 ) after time averaging.

In writing the fluctuation terms, no distinction has been made between the phases. However, it should be noted that the turbulence viscosities of the two phases are generally not equal, and may even be different in the order of magnitude. While the turbulence viscosity of the first phase can be calculated by a $k-\varepsilon \operatorname{model}^{(9)}$, the second phase viscosity is either taken from experiments or calculated from the turbulence behaviour of the first phase $\mathrm{e}^{(10)}$.

\section{3 Coordinate transformation}

For many parabolic flow problems, rectangular Cartesian coordinates were often found to be geometrically inappropriate. Therefore the continuum equations are transformed, making use of the flow streamlines. This permits an automatic adjustment to a domain of physical importance.

First a conversion into a $(X, \psi)$ - coordinate system has to be made (von Mises transformation ${ }^{(11)}$ ) where $\psi$ is a generalized stream function defined as

$$
\frac{\partial \psi}{\partial X}=-\left(\rho \gamma V+\rho \overline{\gamma^{\prime} v^{\prime}}\right)
$$




$$
\frac{\partial \psi}{\partial Y}=\rho \gamma U
$$

which transforms the general equation Eq. ( 5 ) into

$$
\frac{\partial \phi}{\partial X}=-\frac{\partial}{\partial \phi}\left(\rho \gamma \overline{v^{\prime} \phi^{\prime}}\right)+S_{\phi}
$$

A second transformation ${ }^{(3)}$ is performed to obtain a $(X, \omega)$ - coordinate system which makes use of the definition

$$
\omega=\frac{\psi-\psi_{I}}{\psi_{E}-\psi_{I}} .
$$

The stream lines specified by $\phi_{I}$ and $\phi_{E}$ are the stream lines enclosing the flow field of interest in the lateral direction. With $\omega$ for the lateral coordinate, Eq. (11) transforms further to

$$
\begin{aligned}
\frac{\partial \phi}{\partial X}+(a+b w) \frac{\partial \phi}{\partial \omega}= & -\frac{1}{\psi_{E}-\psi_{I}} \frac{\partial}{\partial w}\left(\rho r \overline{v^{\prime} \phi^{\prime}}\right) \\
& +\frac{1}{\rho \gamma U} S_{\phi}
\end{aligned}
$$

The first term represents the convection of $\phi$ in the $X$-direction. The second term accounts for the mass flow rate across a constant $\omega$ - line. The third term is lateral diffusion, and the last term represents various source terms.

\section{4 Discretization, additional equations, and cal- culation procedure}

The general equation, Eq. (13), looks exactly like the corresponding single-phase equation. Therefore, details of the discretization of Eq. (13) will not be discussed here. An extensive presentation is given by Spalding ${ }^{(3)}$. It should be noted however, that the discretization of the momentum equations requires a staggered grid in the lateral direction to provide always reasonable velocity distributions ${ }^{(12)}$. The resulting general finite-difference equation is

$$
d_{i} \phi_{i}=a_{i} \phi_{i+1}+b_{i} \phi_{i-1}+c_{i}
$$

The coefficients $a_{i}$ and $b_{i}$ contain diffusivity and lateral convection at the $i+1 / 2$ and $i-1 / 2$ interfaces of a control volume, respectively. The coefficient $c_{i}$ expresses the effect of convection from the upstream location, as well as the influence of source terms. The coefficient $d_{i}$ counter-balances the effect of diffusivity and convection which are expressed in $a_{i}, d_{i}$, and $c_{i}$. It may also contain parts of the source terms. Besides Eq. (14), other equations have to be provided to enable a calculation of all required variables.

A feature of streamlines is that the flow is always along equi $-\psi$ lines, never across them. This leads to an equation ${ }^{(13)}$ for the local tangents of equi $-\psi$ lines (marked by superscript $\phi$ )

$$
\frac{\rho \gamma V+\rho \overline{\gamma^{\prime} v^{\prime}}}{\rho \gamma U}=\left(\frac{d Y}{d X}\right)^{(\psi)}
$$

Using the transformation formula ${ }^{(3)}$ for $\omega$ gives

$$
\left(\frac{\rho \gamma V+\rho \overline{\gamma^{\prime} V^{\prime}}}{\rho \gamma U}\right)_{i+1 / 2}^{\left(\psi^{\prime}\right)}=\left(\frac{Y-Y_{U}}{\Delta X}\right)_{i+1 / 2}^{(\omega)}
$$

$$
\begin{gathered}
+\left(\phi_{E}-\phi_{I}\right)\left(\frac{a+b \omega}{\rho \gamma U}\right)_{i+1 / 2} \\
\tan \zeta_{i+1 / 2}=\tan \xi_{i+1 / 2}+\left(\frac{\dot{M}^{\prime}}{\rho \gamma U}\right)_{i+1 / 2}
\end{gathered}
$$

The equation is already discretised and written for the control-volume interface $i+1 / 2$. An equivalent equation is valid for the interface location $i-1 / 2$. The gradient, expressed by the down stream location $Y$ and the upstream location $Y_{U}$, is the tangent of an $\omega$ - line (not a $\phi$ - line) which is indicated by the superscript $\omega$. The equation can easily be interpreted. As is shown in Fig. 1, the local tangent of an $\psi$ - line is equal to the local tangent of an $\omega$ - line, taking into account the mass flow $\dot{M}^{\prime}$ which crosses the $\omega$ - line.

A balance of the mass flow for a $(X, \omega)$ - control volume (Fig. 1) leads to

$$
\begin{aligned}
& (\rho \gamma U A)_{i}-(\rho \gamma U A)_{i, U} \\
& \quad-\Delta X\left(\psi_{E}-\psi_{I}\right)\left[(a+b \omega)_{i+1 / 2}\right. \\
& \left.-(a+b \omega)_{i-1 / 2}\right]=0 \\
& (\rho \gamma U A)_{i}-\Omega_{i}\left(\phi_{E}-\psi_{I}\right)_{U} \\
& \quad-\Delta X\left(\dot{M}^{\prime}{ }_{i+1 / 2}-\dot{M}^{\prime}{ }_{i-1 / 2}\right)=0
\end{aligned}
$$

The first two terms are the axial flow in a downstream and an upstream location. The lateral flow crossing the control-volume interfaces is accounted for in the last two terms.

All essential equations are given so far. Other useful equations, dealing with entrainment control, profiles at the boundaries, axial pressure, and others, must be left unnoticed, but can easily be derived similar to equations for single-phase flows ${ }^{(3)}$.

The calculation procedure for iteratively solving the continuum equations is as follows:

first-phase

$$
U_{f} \text { from } X \text {-momentum equation Eq. (14) }
$$

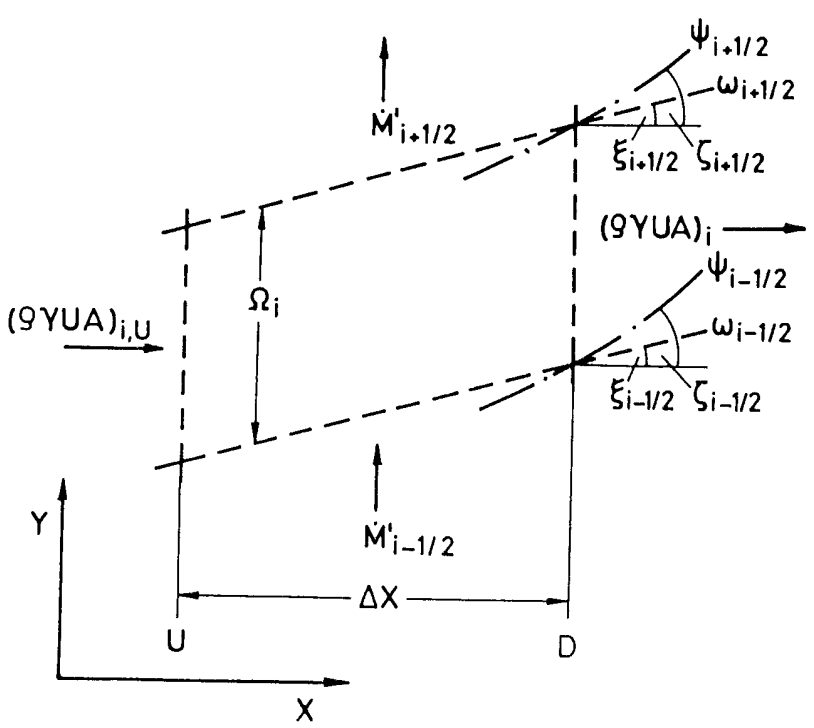

Fig. $1(X, \omega)$ - control volume and mass balance 
$Y_{f}$ from definitions of $\psi_{f}$ and $\omega_{f}$. Eq. $(10 \cdot \mathrm{b})$ and Eq. (12)

$V_{f}$ from tangent of $\psi_{f}$ and $\omega_{f^{-}}$lines. Eq. (16)

$p$ from lateral momentum equation Eq.(14)

$\phi_{f}$ from additional differential equations

second phase

$U_{s}$ from $X$-momentum equation Eq. (14)

$Y_{s}$ from definition of $\psi_{s}$ and $\omega_{s}$. Eq. $(10 \cdot b)$ and Eq. (12)

$V_{s}$ from lateral momentum equation Eq.(14)

$\phi_{s}$ from additional differential equations

1.) Explicit calculation of $\beta$

$Y_{i+1 / 2} Y_{i-1 / 2}$ updating by tangents of $\psi_{s^{-}}$line using $V_{\text {s. }}$.

Eq. $(16 \cdot a)$

$A_{i} /\left(\phi_{E}-\psi_{I}\right)$ updating area $A$ by new $Y^{\prime}{ }_{s}$

Eq. $(16 \cdot \mathrm{b})$

$\beta$ from continuity eq. with new area $A$

$$
\text { Eq. }(17 \cdot a)
$$

2.) Implicit calculation of $\beta$

$\dot{M}^{\prime}{ }_{i+1 / 2} \dot{M}^{\prime}{ }_{i-1 / 2}$ by tangents of $\phi$ and $\omega$

$\beta$ from continuity with new lateral flow

Eq. $(17 \cdot b)$

total mass balance

$\alpha=1-\beta$

Taking into consideration that the continuity equations, momentum equations, $\phi$ - equations, and the definitions of $\omega_{f}$ and $\omega_{s}$ are identically expressed for both phases, enables us to use identical subroutines for both phases. (Source terms may be different but this does not effect the general solution procedure.) Identically calculated are $U_{f}$ and $U_{s} ; Y_{f}$ and $Y_{s} ; \phi_{f}$ and $\phi_{s}$. With little difference $p$ and $V_{s} ; V_{f}$ and $\beta$ (for explicit calculation of $\beta$ ) ; the air volume fraction $\alpha$ is unique.

For the calculation of the volume fraction $\beta$, an explicit and an implicit procedure is outlined. The explicit procedure calculates $\beta_{i}$ by adjusting the coordinates $Y_{i}$ in response to a $(U, V)$ - velocity field and a fixed exact entrainment $a+b \omega$, while the implicit procedure keeps the coordinates $Y_{i}$ fixed per iteration step and adjusts $\beta_{i}$ by varying the entrainment $\dot{M}^{\prime}$. The entrainment $\dot{M}^{\prime}$ is then the exact entrainment plus an apparent entrainment due to a momentary incorrect $Y_{i}$.

2.5 Exchange of variables between the grid of the first- and second-phase.

In the previous chapter it is shown that the introduction of separate grids for each phase leads to similar calculation routines for the two phases, although the calculation sequence is not symmetrical. But the use of two grids makes additional efforts necessary to transmit variables from one grid to the other, since the two grids are not expected to be congruent in the lateral direction, as is shown in Fig. 2. In the downstream $X$ direction, the step length is set to be equal for both grids because of simplicity.

The variables which have to be transferred are the lateral pressure $p$ from the grid of the first phase to the grid of the second phase, the volume fraction $\beta$ from the grid of the second phase to the grid of the first phase to satisfy the global continuity equation, and the exchange of momentum due to interphase friction force.

Because the two grids are usually not congruent, a distinction between the two tasks is made.

1.) A variable has to be transferred from a narrow grid to a wide grid. Then, several control volumes of the narrow grid are combined to establish a representative value for each control volume of the wide grid. These will be called: combining control volumes.

2.) A variable has to be transferred from a wide grid to a narrow grid. Then, a large control volume is split into smaller ones and appropriate values have to be assigned. This will be called: splitting control volumes into sub-domains.

The case of two grids which are only staggered to each other is the boundary case between 1.) and 2.). It is required that formulae for 1.) and 2.) must be identical for this case. Of course, for the trivial case of two congruent grids, the formulae must lead to identical values in both grids.

To perform averaging and control volume splitting, the mass flow is used as a weighting function because the continuum equations are transformed with respect to the mass flow $[(X, \omega)$ - control volumes]. The normalized mass flow per control volume is

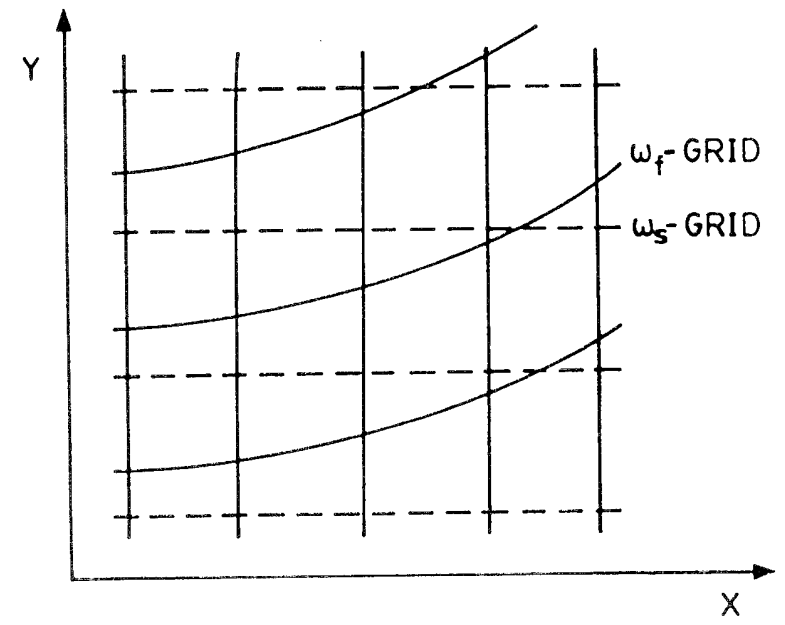

Fig. 2 Not congruent grids for the first - and second phase 


$$
\frac{1}{\phi_{E}-\phi_{I}} \int_{i-1 / 2}^{i+1 / 2} \rho \gamma U d Y=\int_{i-1 / 2}^{i+1 / 2} d \omega .
$$

Within a control volume, the $(\rho \gamma U)$ - profile is constant. Therefore, the integration gives

$$
\frac{(\rho \gamma U)_{i} A_{i}}{\phi_{E}-\psi_{I}}=\omega_{i+1 / 2}-\omega_{i-1 / 2}=\Omega_{i} .
$$

\subsection{Combining control volumes}

In Fig. 3(a) several $i$-control volumes are shown and a representative value $\phi_{j}$ of the $j$-control volume (dashed lines) is sought. Performing an easy summation and averaging of the $\phi_{i^{-}}$values for the control volumes from $k$ till $m$ gives

$$
\phi_{j}=\frac{\Omega_{k} \frac{A_{A}}{A_{A}} \phi_{k}+\Omega_{m} \frac{A_{E}}{A_{m}} \phi_{m}+\sum_{i=k+1}^{m-1} \Omega_{i} \phi_{i}}{\Omega_{k} \frac{A_{A}}{A_{k}}-\Omega_{m} \frac{A_{E}}{A_{m}}+\sum_{i=k+1}^{m-1} \Omega_{i}} .
$$

The first two terms account for the overlapping control volumes marked by cross hatching in Fig. 3 (a), while the third term is a summation over all $\phi_{i}$ control volumes which are entirely within the $\phi_{j}$ control volume. For the overlapping $i$-control volumes Eq. (19) has been made to replace $\Omega_{A}$ and $\Omega_{E}$ by $\Omega_{A}=\Omega_{k} A_{A} / A_{k}$ and $\Omega_{E}=\Omega_{m} A_{E} / A_{m}$.

In the case of an $\omega^{-}$grid in which $\Omega_{i}$ is a constant for all $i$, each control volume contains the same amount of mass flow, and Eq. (20•a) reduces to an expression for an arithmetic mean of $\phi_{j}$

$$
\phi_{j}=\frac{\frac{A_{A}}{A_{k}} \phi_{k}+\frac{A_{E}}{A_{m}} \phi_{m}+\sum_{i=k+1}^{m-1} \phi_{i}}{\frac{A_{A}}{A_{k}}+\frac{A_{E}}{A_{m}}+m-k-2}
$$

with area weighting at the edge control volumes.

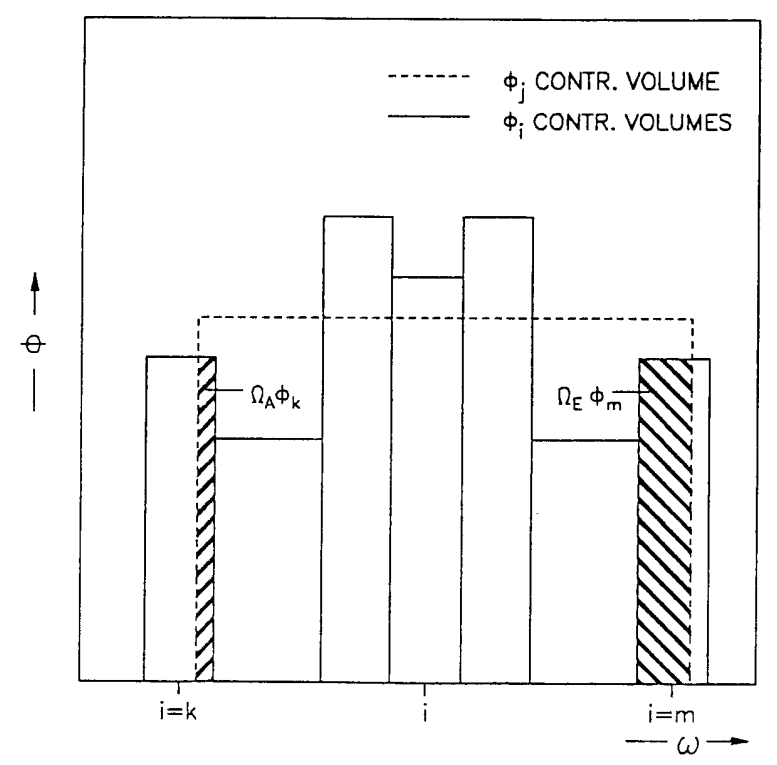

(a) Control volume averaging

\subsection{Splitting control volumes into} sub-domains

Two control volumes $i$ and $i+1$ which have to be split into sub-domains $j$ and $j+1$ are shown in Fig. 3(b). Two different cases are considered. First, the sub-domain $j+1$ maintains throughout the control volume $j+1$. Then the control volume value $\phi_{i+1}$ is assigned to the sub-domain, which gives

$$
\phi_{j+1}=\phi_{i+1} \text {. }
$$

Second, the sub-domain $j$ overlaps the controlvolume boundary of $i$, as shown in Fig. 3(b) by the cross hatched areas. Then an average value $\phi_{j}$ is calculated using an equation similar to Eq. $(20 \cdot a)$

$$
\phi_{j}=\frac{\Omega_{i} \frac{A_{A}}{A_{i}} \phi_{i}+\Omega_{i+1} \frac{A_{E}}{A_{i+1}} \phi_{i+1}}{\Omega_{i} \frac{A_{A}}{A_{i}}+\Omega_{i+1} \frac{A_{E}}{A_{i+1}}} .
$$

For an equi-distant grid in $\omega$ the mass weighted Eq. $(21 \mathrm{~b})$ reduces to an equation with area weighting

$$
\phi_{j}=\frac{\frac{A_{A}}{A_{i}} \phi_{i}+\frac{A_{E}}{A_{i+1}} \phi_{i+1}}{\frac{A_{A}}{A_{i}}+\frac{A_{E}}{A_{i+1}}} .
$$

\subsubsection{Advantages of the method of variable} exchange

The presented method is of course not the only possible method for an interpolation of variables between grid nodes. One of the alternatives is cubic splines. Although splines ensure that variables are exactly approximated at their grid location, overshooting may occur for the interpolation values. A conservation criteria which requires that the total convective flux must be a constant and independent of the control volume distribution can hardly be satisfied.

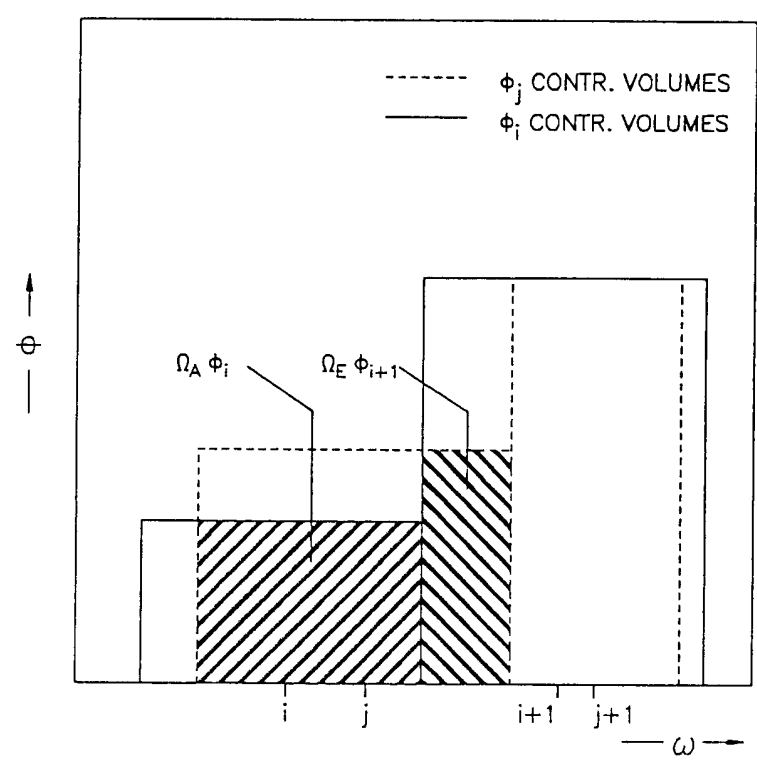

(b) Splitting control volumes into sub-domains

Fig. 3 
For two different control volume distributions $i$ and $j$ it will be in general

$$
\sum_{i=k}^{m} \Omega_{i} \phi_{i} \neq \sum_{j=1}^{n} \Omega_{j} \phi_{j}
$$

Besides this, the prediction of cubic spline functions increases the computational expenditures considerably. Each variable which has to be interpolated and transferred requires its own set of spline functions, with as many as nearly 4-times the number of unknown coefficients than the number of grid intervals. In contrast, the method of variable exchange presented in the previous chapters does not have these shortcomings. Its advantages are:

The presented formulae are very simple and fast to compute.

Ratios of overlapping areas like $A_{A} / A_{i+1}$ and $A_{E} /$ $A_{i}$ (Fig. 3(a) and Fig. 3(b)) have to be calculated only once per iteration (or even once per step in the $X$-direction) and are then valid for all variables which have to be transferred.

A conservation criterion $\sum_{i=k}^{m} \Omega_{i} \phi_{i}=\sum_{j=1}^{n} \Omega_{j} \phi_{j}$ is satisfied ( $\Omega_{i}$ and $\Omega_{j}$ belonging to the same phase but different grids). If at least one sub-domain is entirely within a control volume, the $\phi_{i}$ value is at least represented by one $\phi_{j}$ value, which has the advantage of preserving minimum and maximum values of $\phi_{i}$ also in the $j$-grid.

The Eqs. (20),(21) lead to the same result for a $j$ grid which is just staggered in the $i$-grid. For a congruent $i$ - and $j$ - grid the equations vanish and the values of $\phi$ are identical in both grids.

\section{Applications of the Calculation Procedure and Discussion.}

To estimate the effect of false diffusion on the second phase, an extreme case is examined. A circular two-phase jet emerges into a stagnant surrounding and contains a second phase which is considered as being purely convective with no diffusivity, no extra forces, and no interphase friction. This must lead to zero spreading and to no change in the velocity and volume fraction profiles of the second phase for downstream locations. As starting conditions, the axial velocity profiles of both phases are set to be uniform at $30 \mathrm{~m} / \mathrm{s}$. The lateral velocity is zero. The volume fraction of the second phase has also a uniform starting profile and has been set to $\beta=10^{-3}$. The first phase is calculated by balancing axial convection, lateral entrainment and diffusion (Eq. (13)) but omitting any interphase forces and extra forces.

Figure 4 shows velocity profiles of the first and second phase for distances up to $0.5 \mathrm{~m}$ from the outlet. While the firstphase spreads strongly further down- stream (computational grid is marked by + ), the second-phase keeps its velocity and shows no sign of spreading, as must be expected for a purely convective flow. It is obvious that a similar excellent result can hardly be obtained by a computational code where the second-phase is calculated in the strongly spreading grid of the first phase. Some amount of false diffusion is unavoidable, even by using a refined grid and/or a more sophisticated scheme.

Besides this simple test case which clearly confirms the advantage of using two independent grids, a turbulent two-phase jet is calculated and compared to measured results which have been presented by authors ${ }^{(14)(15)}$. Unlike the previous test case, particle diffusivity, gravity and drag force are accounted for. The particles have an average diameter of $d_{p}=64 \mu \mathrm{m}$ and a density of $2590 \mathrm{~kg} / \mathrm{m}^{3}$. The outlet velocity is $30 \mathrm{~m} / \mathrm{s}$ and the mass loading ratio $M$ $=0.3$. The turbulent quantities are calculated by a $k-\varepsilon$ model, and the particle diffusivity is drawn from the experimental data.

The calculation is performed twice to compare the performance of the implicit and explicit calculation of the volume fraction. No differences in results are found ; only slightly faster convergence behaviour of the calculation procedure using the explicit formulae. While the implicit formulae requires on the average 3-4 cycles per $X$ - step, the explicit formulae requires only 2 cycles to achieve the desired accuracy. The iteration ends if a change in $\beta$ is less than $0.1 \%$ of the volume fraction at the center line.

In the main stream direction the step length is calculated to $10 \%$ of the width of the particle phase. In

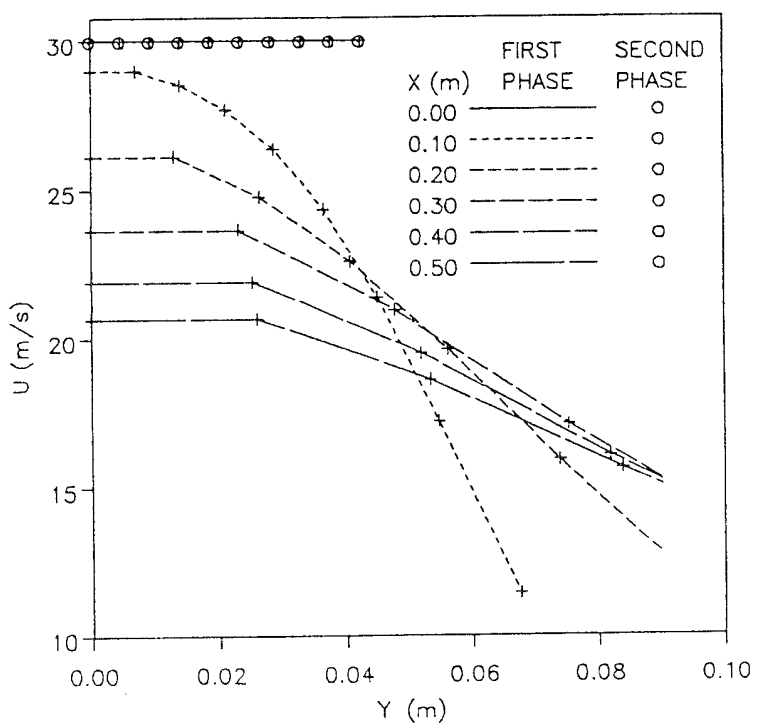

Fig. 4 Lateral velocity profiles of a two-phase jet with no diffusion and extra forces for the second phase 
the lateral direction, 30 grid points are used for the particle grid and the air grid. The distribution of the control volumes in the lateral direction are shown in Fig. 5(a) for $X / D=20$ from the outlet. On the right side of the center line the stepwise profile of the air velocity $U$ in the air grid is shown. On the left side, the same velocity distribution is shown but it is transferred into the particle-phase grid. It can be seen that the velocity profile is well preserved in the particle grid, which confirms the applicability of Eqs. (20) and (21).

In Fig. 5(b) the same control volumes are shown, except for the particle velocity $U_{p}$ which is calculated in the particle grid and then transferred into the air

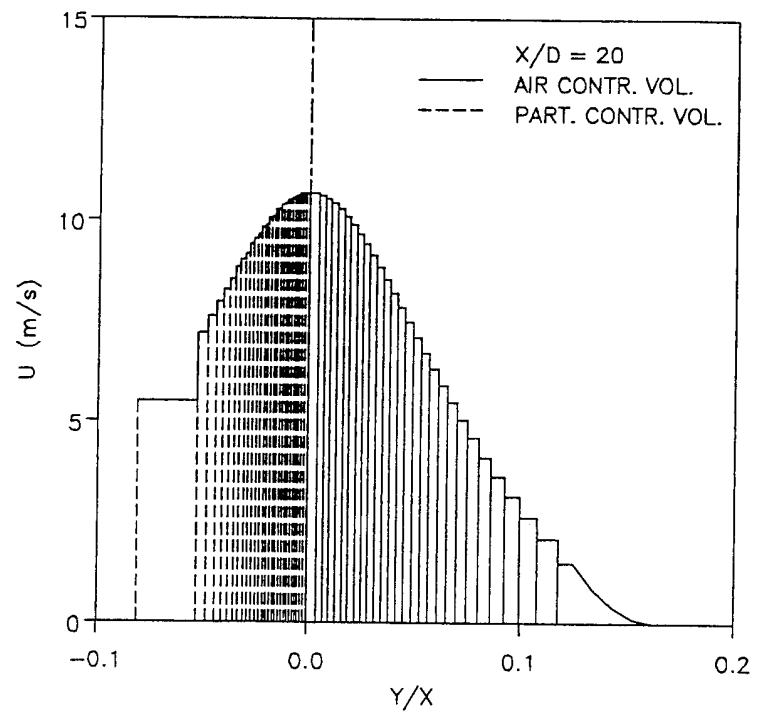

(a) Air velocity distribution shown for the air and particle control volumes

grid. Also, here, the transfer formulae have no effect on the velocity profile of the particles in the air grid. The large particle control volume at the edge of the particle phase is split into several sub-domains by the air grid, taking accurate account of the particle velocity but not of the gradient. This does not affect the present calculation since the gradient of the lateral pressure and the volume fraction (Eq. (3) and Eq. $(10 \cdot a))$ which are shared by both phases are small. However, to take correct account of gradients in other cases, not only the variable itself should be transferred between the grids, but also its gradient. This leads to a variable and a gradient distribution

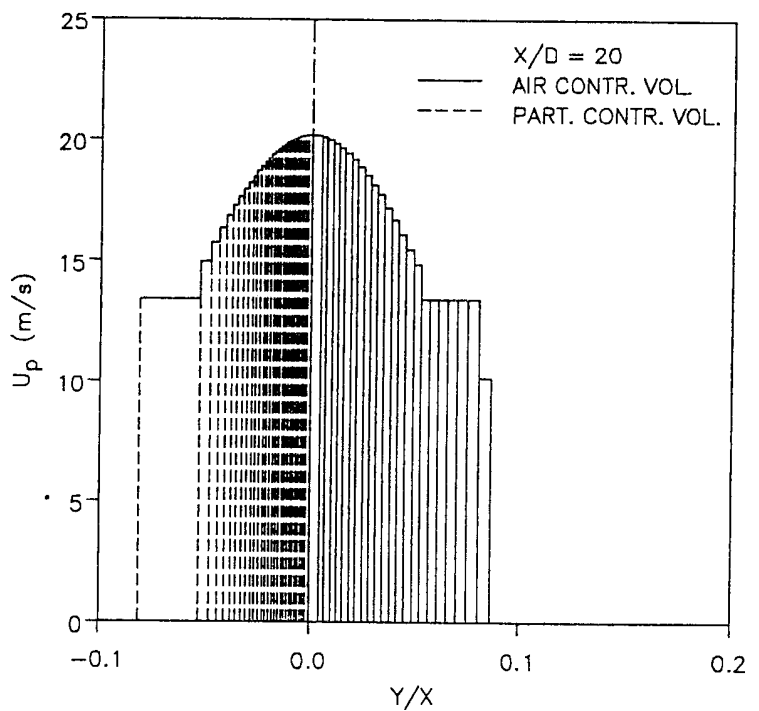

(b) Particle velocity distribution shown for the particle and air control volumes

Fig. 5

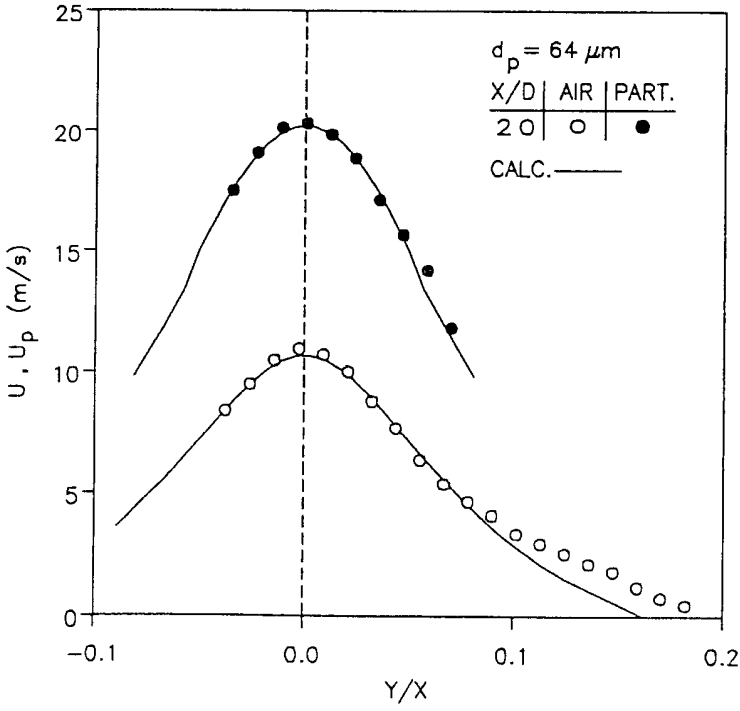

Fig. 6 Comparison between calculated and measured velocity profiles for a partjcle laden free jet

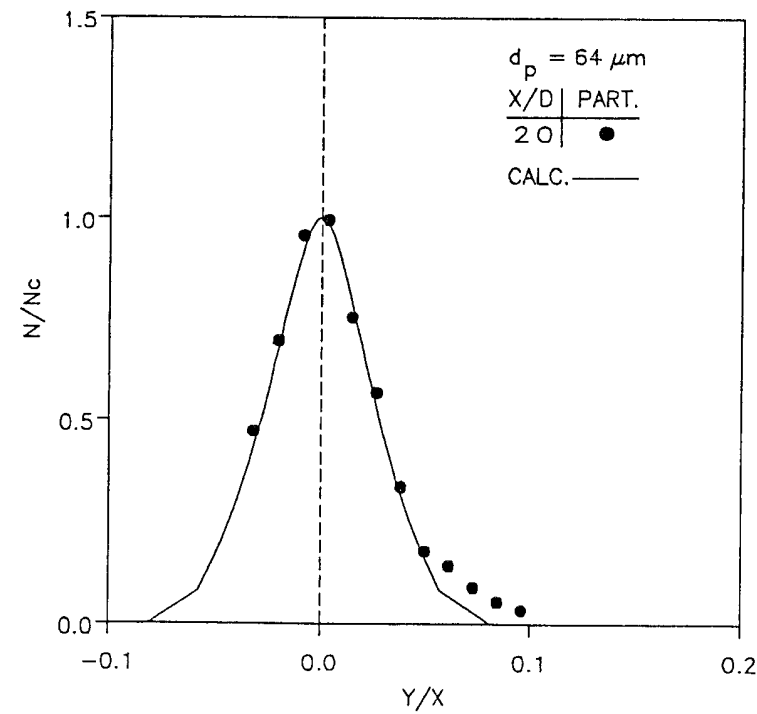

Fig. 7 Comparison between calculated and measured number densities for a particle laden free jet 
which do not strongly correspond to each other, but will correctly account for gradient terms in the continuum equations.

Figure 6 shows the agreement between calculated and experimental results for the main stream velocities at the axial location $X / D=20$ from the outlet. The measuring technique of velocity in two-phase flow and experimental results on flow characteristics of circular jet were presented in reference ${ }^{(14)}$, and best fitting functions of lateral mean velocity and number density profiles for the same flow condition were obtained in the report by the authors ${ }^{(15)}$. The agreement is good, except for the air-velocity at the jet edge region. There the measured values are somewhat higher than the calculated results. The discrepancy is believed to be due to a bias error ${ }^{(16)}$ in the measurements rather than to inaccuracies in the calculation or physical modeling. The number density which is sensitive to false diffusion is shown in normalized form in Fig. 7. Also here the calculation is in good accordance with the measurements.

\section{Conclusion}

a) The present paper has described a generally applicable, accurate and economical method for the calculation of dispersed, parabolic and turbulent twophase flows.

b) Convection, diffusion and viscous action, as well as entrainment and volume fraction fluctuations are taken into account for the second phase also.

c) The incorporation of independent computational grids for each phase is a feature which reduces false diffusion to a minimum.

d) It also enables us to use nearly identical routines for both phases since their continuum equations are similar.

e) Formulae for the transfer of variables between the grid of the first phase and the grid of the second phase are proven to perform satisfactorily.

\section{References}

(1) Patankar, S. V. and Spalding, D. B., Heat and
Mass Transfer in Boundary Layers (2nd ed.), Intertext Books, (1970).

(2) Patankar, S. V. and Spalding, D. B., A Calculation Procedure for Heat, Mass and Momentum Transfer in Three-Dimensional Parabolic Flows, Int. J. Heat Mass Transf. Vol. 15, No. 10 (1972), p. 1787.

(3) Spalding, D. B., GENMIX: A General Computer Program for Two-Dimensional Parabolic Phenomena, (1977), Pergamon Press.

(4) Spalding, D.B., Numerical Computation of Multiphase Flows, Lecture Notes, Purdue Univ., (1979).

(5) Sha, W.T. and Soo, S.L., Multidomain Multiphase Fluid Mechanics, Int. J. Heat Mass Transf., Vol. 21, No. 12 (1978), p.1581.

(6) Anderson, D. A., Tannehill, J. C. and Pletcher, R. H., Computational Fluid Mechanics and Heat Transfer, (1984), McGraw-Hill.

( 7 ) Soo, S. L., Fluid Dynamics of Multiphase Systems, Blaisdell, Waltham, (1967).

(8) Launder, B. E., in Turbulence (ed. P. Bradshaw), (1976), p. 231, Springer Verlag.

(9) Elghobashi, S. E. and Abou-Arab, T. W., A Two -Equation Turbulence Model for Two-Phase Flows, Phys. Fluids, Vol. 26 No. 4 (1983), p. 931.

(10) Peskin, R. L., Stochastic Estimation Appl. to Turbulent Diffusion, Int. Symp. on Stochastic Hydraulics, (ed. Chiu, C. L.), University of Pitsburgh, Pitsburgh, Pa., (1971), p. 251.

(11) Schlichting, H., Boundary Layer Theory, (1979), McGraw-Hill.

(12) Patankar, S. V., Numerical Heat Transfer and Fluid Flow, (1980), McGraw-Hill.

(13) Lu, P.-C., Introduction to the Mechanics of Viscous Fluids, (1977), McGraw-Hill.

(14) Hishida, K., Kaneko, K. and Maeda, M., Turbulence Structure of Gas-Solids Two-Phase Circular Jet, (in Japanese), Trans. Jpn. Soc. Mech. Eng., Vol. 51 No. 467, B, (1985), p. 2330.

(15) Fleckhaus, D., Hishida, K. and Maeda, M., Effects of Laden Solid Particles on the Turbulent Flow Structure of a Round Free Jet, Experiments in Fluids, Vol. 5, No. 5 (1987), p. 323.

(16) Durst, F., Meiling, A. and Whitelaw, J. H., Principles and Practice of Laser-Doppler Anemometry, (1976), Academic Press. 